\title{
UTILIZAÇÃO DA CALORIMETRIA EXPLORATÓRIA DIFERENCIAL (DSC) PARA AVALIAÇÃO DA DESNATURAÇÃO TÉRMICA DE DIFERENTES COLÁGENOS BOVINOS
}

\author{
A. P. KEMPKA ${ }^{1}$, M. M. P. ANDRADE ${ }^{2}$, F. B. MELLO ${ }^{3}$, W. S. WEIGELT ${ }^{3}$, G. WALTER ${ }^{3}$ \\ M. B. PINTON ${ }^{3}$, M. A. MAZUTTI ${ }^{4}$ I. M. DEMIATE ${ }^{2}$ e R. C. PRESTES ${ }^{3 *}$
${ }^{1}$ Universidade do Estado de Santa Catarina, Departamento de Engenharia de Alimentos
${ }^{2}$ Universidade Estadual de Ponta Grossa, Departamento de Engenharia de Alimentos
3* Universidade Federal de Santa Maria, Departamento de Tecnologia e Ciência dos Alimentos
${ }^{4}$ Universidade Federal de Santa Maria, Departamento de Engenharia Química
*E-mail para contato: rosacrisprestes@ @otmail.com

\begin{abstract}
RESUMO - A partir do colágeno são obtidos o colágeno hidrolisado, a fibra de colágeno e a gelatina. $\mathrm{O}$ objetivo deste estudo foi avaliar a desnaturação térmica de 18 diferentes amostras de colágenos bovinos (amostras in natura e hidrolisadas enzimaticamente com e sem o emprego concomitante de ultrassom) utilizando a técnica de Calorimetria Exploratória Diferencial (DSC). Foi utilizada a faixa de aquecimento de $-10{ }^{\circ} \mathrm{C}$ a $100{ }^{\circ} \mathrm{C}$ e cadinhos de alumínio previamente selados. Observaram-se diferenças significativas $(\mathrm{p}<0,05)$ na temperatura de pico $\left(\mathrm{T}_{\mathrm{p}}\right)$ e entalpia $(\Delta \mathrm{H})$ para as amostras avaliadas. $\mathrm{T}_{\mathrm{p}}$ variou de $18,90{ }^{\circ} \mathrm{C}$ a $58,13{ }^{\circ} \mathrm{C}$ e $\Delta \mathrm{H}$ de $2,7 / \mathrm{J} \mathrm{g}^{-1}$ a $1153,00 / \mathrm{J}_{\mathrm{g}} \mathrm{g}^{-1}$. Os maiores valores de $\mathrm{T}_{\mathrm{p}}$ e $\Delta \mathrm{H}$ foram encontrados para a fibra de colágeno e gelatina in natura em decorrência da estrutura mais íntegra. As amostras submetidas ao ultrassom apresentaram menores valores de $T_{p}$ e $\Delta$ H. A avaliação da desnaturação térmica pode auxiliar na caracterização e seleção destes colágenos para as mais diversas aplicações industriais.
\end{abstract}

\section{INTRODUÇÃO}

O colágeno é a proteína dominante no tecido conjuntivo sendo encontrado sob várias formas em tecidos de todas as espécies de organismos multicelulares, exercendo diversas funções dependendo de sua localização (Shimokomaki et al., 2006; Damoradan et al., 2010).

A partir do colágeno nativo podem ser obtidos: fibra de colágeno, colágeno 
parcialmente hidrolisado (gelatina) e colágeno hidrolisado. Para fins de produção industrial, a gelatina é obtida do colágeno através da hidrólise ácida ou alcalina. $\mathrm{Na}$ extração ácida a gelatina obtida é classificada como Tipo A, apresenta ponto isoelétrico entre 7 e 9 e nesse processo ocorre a reorganização física da estrutura e mínimas alterações hidrolíticas resultando em ampla faixa de distribuição de massa molar. $\mathrm{Na}$ hidrólise alcalina o produto é denominado gelatina Tipo $\mathrm{B}$, apresenta ponto isoelétrico entre 4,7 e 5,5, e este processo é mais drástico, hidrolisa até aminoácidos o que resulta em menor faixa de distribuição de massa molar (Scrieber \& Gareis, 2007; Ockerman \& Hansen, 1994; Deman, 1999; Damoradan et al., 2010).

O colágeno hidrolisado é obtido por hidrólise química e enzimática sob condições controladas (Scrieber \& Gareis, 2007). A diferença entre o colágeno hidrolisado e a gelatina é que o colágeno hidrolisado dissolve-se em água ou salmoura e a grande maioria não apresenta capacidade de geleificação (Damoradan et al., 2010). A fibra de colágeno é obtida das camadas internas do couro bovino através de processo químico (tratamento alcalino com hidróxido de cálcio), segue para o desengorduramento e secagem a baixas temperaturas (processo menos drástico) (Santana et al., 2012; Máximo \& Cunha, 2010). A fibra de colágeno em pó é obtida por processo similar, porém submetida a temperaturas mais elevadas e posterior moagem.

O objetivo deste estudo foi avaliar a desnaturação térmica de 18 diferentes amostras de colágenos bovinos (amostras in natura e hidrolisadas enzimaticamente com e sem o emprego concomitante de ultrassom), utilizando a técnica de Calorimetria Exploratória Diferencial (DSC).

\section{MATERIAIS E MÉTODOS}

Os experimentos foram realizados nos Laboratórios de Engenharia Química da Universidade Federal de Santa Maria (Santa Maria, RS) e nos Laboratórios de Engenharia de Alimentos da Universidade Estadual de Ponta Grossa (Ponta Grossa, PR).

Foram testadas 18 diferentes amostras de colágeno bovino (6 amostras in natura e 12 amostras hidrolisadas enzimaticamente com e sem o emprego concomitante de ultrassom). As amostras denominadas in natura foram: a fibra natural de colágeno (FNC) (Novaprom Food Ingredients, Lins, SP), fibra de colágeno em pó (FP) (Novaprom Food Ingredients, Lins, SP), colágeno parcialmente hidrolisado 1 ou gelatina 1 (GEL1) (Gelita South America, Maringá, PR), colágeno parcialmente hidrolisado 2 ou gelatina 2 (GEL2) (Gelatina, Rousselot Gelatinas do Brasil Ltda., São Paulo, SP) colágeno hidrolisado 1 (HID1) (Peptiplus, Gelita South America, Maringá, 
PR) e colágeno hidrolisado 2 (HID2) (Peptan B, Rousselot Gelatinas do Brasil Ltda., São Paulo, SP).

As amostras de colágeno in natura foram hidrolisadas enzimaticamente com e sem o emprego de ultrassom concomitantemente a hidrólise. Foi utilizada a enzima Alcalase 2.4L® (Tovanni Benzaquen Ingredientes, endopeptidase de Bacillus licheniformis com $2.4 \mathrm{AU} / \mathrm{g}$ ). A reação enzimática foi conduzida à temperatura e $\mathrm{pH}$ de máxima atividade catalítica da enzima, $55^{\circ} \mathrm{C}$ e pH 7,5 de acordo com a literatura e seguindo a metodologia utilizada por Schmidt \& Salas-Mellado (2009), com algumas modificações. O substrato foi homogeneizado em uma solução tampão fosfato $0,2 \mathrm{M}$. Pesou-se uma determinada quantidade de colágeno que correspondeu a uma concentração total de proteína em solução de $5 \%(\mathrm{~m} / \mathrm{v})$. A enzima foi adicionada ao meio variando-se sua concentração em relação à massa total de proteínas de $8,0 \%$ (g de enzima/g proteína). Para as amostras que foram hidrolisadas com ultrassom foi utilizado banho ultra-sônico (Modelo USC - 1800A, UNIQUE). A potência utilizada foi de 132 W e frequência de $40 \mathrm{KHz}$ ao mesmo tempo em que ocorreu a hidrólise enzimática.

As curvas DSC foram obtidas em um equipamento DSC-Q200 (TA-Instruments, $E U A$ ), o qual foi previamente calibrado com índio de alto grau de pureza de 99,99\%, pf $=156,6{ }^{\circ} \mathrm{C}, \Delta \mathrm{H}=28,56 \mathrm{~J}_{\mathrm{g}} \mathrm{g}^{-1}$.Para registrar a entalpia e as temperaturas do processo de desnaturação das amostras, foi utilizado os seguintes parâmetros: fluxo de ar de $50 \mathrm{~mL}$ $\min ^{-1}$, razão de aquecimento de $10{ }^{\circ} \mathrm{C} \min ^{-1}$, a faixa de aquecimento foi de -10 a $100{ }^{\circ} \mathrm{C}$, foram pesadas cerca de 3,0 mg de colágeno, que foi misturado com água deionizada em uma proporção de 6:1 (água: colágeno, $\mathrm{m} / \mathrm{m}$ ) e a mistura foi mantida em repouso durante 120 minutos sob refrigeração $\left(7^{\circ} \mathrm{C}\right)$ a fim de equilibrar o teor de umidade e hidratarem. A realização da análise foi com cadinhos de alumínio selado.

O delineamento experimental foi casualizado e foram realizadas duas repetições para cada teste. Os resultados foram submetidos à Análise de Variância - ANOVA e Teste de Tukey com nível de significância $(\mathrm{p}<0,05)$ utilizando o programa Statistica ${ }^{\circledR}$ 9.0 (STATSOFT, INC).

\section{RESULTADOS E DISCUSSÃO}

A DSC é comumente utilizada para avaliar a estabilidade e desnaturação térmica do colágeno e seus derivados. Foram mensuradas as temperaturas de ocorrência das alterações decorrentes da desnaturação do colágeno: temperatura inicial $\left(\mathrm{T}_{0)}\right.$, temperatura de pico $\left(\mathrm{T}_{\mathrm{p})}\right.$, temperatura de conclusão $\left(\mathrm{T}_{\mathrm{c}}\right)$ e a entalpia da reação

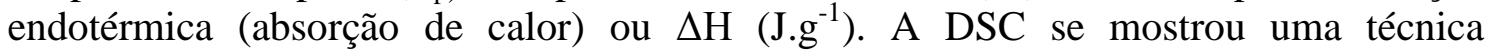
adequada para avaliação da desnaturação térmica para 10 das 18 amostras de colágeno testadas. Os resultados podem ser observados na Tabela 1. Algumas amostras após a hidrólise com ou sem ultrassom não apresentaram picos nem entalpia de desnaturação, 
pois já se encontravam hidrolisadas completamente ou não apresentaram a transição de gel para sol.

Tabela 1 - Resultados de DSC de desnaturação para as diferentes amostras de colágeno testadas.

\begin{tabular}{ccccc}
\hline & \multicolumn{4}{c}{ DSC de desnaturação* } \\
\cline { 2 - 5 } Amostras & $T o /{ }^{\circ} \mathrm{C}$ & $T p /{ }^{\circ} \mathrm{C}$ & $T c{ }^{\circ} \mathrm{C}$ & $\Delta H / \mathrm{J}^{-\mathrm{g}^{-1}}$ \\
\hline FNC in natura & $22,2 \pm 0,55^{\mathrm{c}}$ & $41,49 \pm 0,01^{\mathrm{b}}$ & $57,34 \pm 0,17^{\mathrm{b}}$ & $605,76 \pm 9,11^{\mathrm{b}}$ \\
FNC hidrolisada com US & $3,62 \pm 0,37^{\mathrm{i}}$ & $14,9 \pm 0,17^{\mathrm{h}}$ & $21,84 \pm 0,15^{\mathrm{i}}$ & $52,69 \pm 2,96^{\mathrm{e}}$ \\
FNC hidrolisada sem US & $6,59 \pm 0,16^{\mathrm{g}}$ & $18,54 \pm 0,28^{\mathrm{g}}$ & $33,40 \pm 0,05^{\mathrm{f}}$ & $95,04 \pm 3,95^{\mathrm{d}}$ \\
FP in natura & $36,29 \pm 0,23^{\mathrm{a}}$ & $41,82 \pm 0,16^{\mathrm{b}}$ & $44,44 \pm 0,08^{\mathrm{c}}$ & $2,70 \pm 0,26^{\mathrm{h}}$ \\
GEL1 in natura & $26,47 \pm 0,03^{\mathrm{b}}$ & $58,13 \pm 0,01^{\mathrm{a}}$ & $69,83 \pm 0,04^{\mathrm{a}}$ & $1153,0 \pm 4,35^{\mathrm{a}}$ \\
GEL1 hidrolisada sem US & $26,53 \pm 0,04^{\mathrm{b}}$ & $29,43 \pm 0,02^{\mathrm{c}}$ & $34,12 \pm 0,14^{\mathrm{e}}$ & $17,44 \pm 1,0^{\mathrm{g}}$ \\
GEL2 in natura & $16,37 \pm 0,01^{\mathrm{e}}$ & $23,51 \pm 0,01^{\mathrm{f}}$ & $28,60 \pm 0,03^{\mathrm{h}}$ & $102,6 \pm 0,98^{\mathrm{d}}$ \\
GEL2 hidrolisada com US & $20,8 \pm 0,01^{\mathrm{d}}$ & $25,26 \pm 0,01^{\mathrm{e}}$ & $29,89 \pm 0,02^{\mathrm{g}}$ & $19,80 \pm 0,17^{\mathrm{g}}$ \\
HID1 in natura & $4,47 \pm 0,16^{\mathrm{h}}$ & $25,76 \pm 0,02^{\mathrm{d}}$ & $36,17 \pm 0,02^{\mathrm{d}}$ & $200,23 \pm 6,51^{\mathrm{c}}$ \\
HID1 hidrolisado sem US & $8,47 \pm 0,31^{\mathrm{t}}$ & $15,11 \pm 0,01^{\mathrm{h}}$ & $21,39 \pm 0,03^{\mathrm{j}}$ & $37,56 \pm 0,88^{\mathrm{f}}$
\end{tabular}

(*) $\mathrm{T}_{\mathrm{o}}$ "onset" temperatura inicial, Tp temperatura de pico, $\mathrm{T}_{\mathrm{c}}$ "endset" temperatura de conclusão, $\Delta \mathrm{H}$ entalpia de desnaturação

(**) Médias seguidas de uma mesma letra na coluna não diferem significativamente pelo teste de Tukey $(\mathrm{p}>0,05)$.

(***) FNC corresponde a: Fibra Natural de Colágeno, FP corresponde a Fibra de Colágeno em pó, GEL1 corresponde a Gelatina 1, GEL2 corresponde a Gelatina 2 e HID corresponde ao Colágeno Hidrolisado 1.

A temperatura inicial $\left(\mathrm{T}_{0}\right)$ variou entre 3,62 a $26,53{ }^{\circ} \mathrm{C}$, a temperatura de pico $\left(\mathrm{T}_{\mathrm{p}}\right)$ de 14,9 a $58,13{ }^{\circ} \mathrm{C}$ e a temperatura de conclusão $\left(\mathrm{T}_{\mathrm{c}}\right)$ de 21,39 a $57,34{ }^{\circ} \mathrm{C}$ para as amostras avaliadas. Os maiores valores de entalpia $(\Delta \mathrm{H})$ foram encontrados para a GEL1 e FNC ambas in natura (1153,00 e 605,76 J.g ${ }^{-1}$ respectivamente). Menores valores de entalpia foram encontrados para as amostras de FP in natura $\left(2,70 \mathrm{~J}_{\mathrm{g}}{ }^{-1}\right) \mathrm{e}$ GEL1 hidrolisada sem ultrassom. Quando comparados os resultados de entalpia e temperaturas para a amostra de FNC percebeu-se que houve redução das temperaturas e 
entalpia em decorrência do processo de hidrólise, e quando a hidrólise foi combinada com ultrassom os valores foram ainda menores. Estes resultados podem ser justificados pela estrutura mais íntegra da FNC e da GEL1.

Segundo Torley et al. (2000), a molécula do colágeno é constituída de três cadeias polipeptídicas (duas $\alpha_{1}$ e uma $\alpha_{2}$ ) sendo que em geral a estrutura contém cerca de $30 \%$ de glicina, $12 \%$ de prolina, $11 \%$ de alanina, $10 \%$ de hidroxiprolina, $1 \%$ de hidroxilisina e pequenas quantidades de aminoácidos polares e carregados (Scrieber \& Gareis, 2007; Deman, 1999; Damoradan et al., 2010). A estabilidade térmica do colágeno está relacionada com seu conteúdo de aminoácidos (prolina e hidroxiprolina). Quanto mais elevado o conteúdo de aminoácidos maior é a estabilidade das hélices. O colágeno se desnatura a temperaturas superiores gerando uma mescla de espécies com uma, duas ou três cadeias polipeptídicas enroladas ao acaso (Wong, 1995). Pode-se verificar indiretamente que algumas das amostras analisadas perderam a estrutura secundária (hélice) e que houve diferenças entre a FNC, FP, GEL1, GEL2 e HID1, mostrando que há influência do processo de extração e comprovando que a hidrólise enzimática com ou sem ultrassom afetou a estrutura e características destes colágenos.

Li et al. (2009) relataram que a utilização de ultrassom permitiu aumentar o rendimento e diminuir o tempo de processo de extração de colágeno de tendão bovino. Segundo estes autores há um aumento da dissolução do substrato, permitindo uma maior dispersão e abertura das fibrilas de colágeno facilitando a ação da enzima.

Rochdi et al. (2000) mencionaram temperaturas de desnaturação de colágeno de epimísio e colágeno intramuscular entre 58,6 e $61,6^{\circ} \mathrm{C}$ e entalpia (J/g de amostra seca)

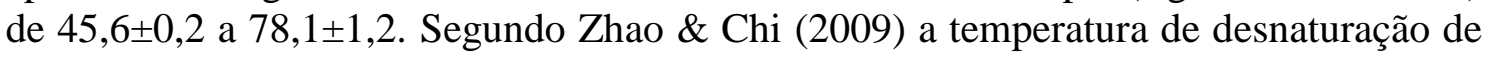
colágeno da pele de suínos e bovinos está entre 60 e $65{ }^{\circ} \mathrm{C}$. Outros estudos mencionaram temperaturas de desnaturação do colágeno variando entre $30^{\circ} \mathrm{C}$ e $60{ }^{\circ} \mathrm{C}$, dependendo da origem e tipo de colágeno testado, sendo evidenciadas menores temperaturas para os colágenos de pescados e maiores para os de origem bovina e suína (Liu et al., 2014 e Sinthusamran et al., 2013).

A presença de aminoácidos, particularmente a hidroxiprolina e prolina, contribui para a estabilidade da tripla hélice através das pontes de hidrogênio entre as cadeias $\alpha$. As variações nas temperaturas $\left(T_{0}, T_{p}\right.$ e $\left.T_{c}\right)$ entre os colágenos, podem ser correlacionadas com o conteúdo de aminoácidos e com os processos de extração e hidrólise. Menores temperaturas de desnaturação indicam menor estabilidade e este é um dos fatores que afetaria a aplicação posterior destes colágenos. A redução da estabilidade está diretamente relacionada ao conteúdo de prolina e hidroxiprolina (Veeruraj et al., 2013; Nagai et al., 2008; He et al., 2012; Wang et al., 2014; Kaewdang et al., 2014 e Pati et al., 2010). 
A utilização da DSC permitiu avaliar a desnaturação térmica de $56 \%$ das amostras de colágeno testadas sendo mais adequada para as amostras de colágeno com estrutura intacta ou mais próxima do colágeno natural e com maior massa molar. Como era esperado, após a hidrólise, algumas amostras não apresentaram picos devido da perda da propriedade de transição de gel para sol.

As amostras submetidas ao tratamento de ultrassom concomitantemente com a hidrólise enzimática foram as que apresentaram menor entalpia e menores temperaturas, comprovando-se os efeitos da sonicação em proporcionar maior contato enzimasubstrato e maior rendimento do processo (maior grau de hidrólise).

Com base nos resultados obtidos pode-se concluir que a DSC é uma alternativa de técnica complementar para os processos de avaliação, caracterização e discriminação (origem bovina, suína ou de aves ou ainda colágenos in natura ou hidrolisados) de diferentes tipos de colágeno. Também pode ser empregada para a detecção de fraudes e no estudo do comportamento dos colágenos em determinadas condições que se aproximam das condições a que estes são submetidos a nível industrial.

\section{CONCLUSÕES}

Os resultados da DSC permitiram avaliar a estabilidade e desnaturação térmica dos diferentes tipos de colágeno bovinos testados e mostraram diferenças significativas $(\mathrm{p}<0,05)$ na temperatura de pico $\left(\mathrm{T}_{\mathrm{p}}\right)$ e entalpia $(\Delta \mathrm{H}) . \mathrm{T}_{\mathrm{p}}$ variou de $18,90{ }^{\circ} \mathrm{C}$ a $58,13{ }^{\circ} \mathrm{C}$ e $\Delta \mathrm{H}$ de $2,7 / \mathrm{J}_{\mathrm{g}} \mathrm{g}^{-1}$ a $1153,00 / \mathrm{J}_{\mathrm{g}}{ }^{-1}$. Os maiores valores de $\mathrm{T}_{\mathrm{p}}$ e $\Delta \mathrm{H}$ foram encontrados para a fibra de colágeno e gelatina 1 in natura, em decorrência da estrutura mais íntegra destes colágenos. As amostras submetidas ao ultrassom apresentaram menores valores de $T_{p}$ e $\Delta H$. A avaliação da desnaturação térmica pode auxiliar na caracterização e seleção destes colágenos para as mais diversas aplicações industriais tais como: produtos biomédicos e farmacêuticos, indústria de cosméticos, encapsulamento, produção de embalagens biodegradáveis e na área de alimentos como agente gelificante e espumante.

\section{REFERÊNCIAS}

DAMODARAN, S.; PARKIN, K. L.; FENNEMA, O.R.; Química de Alimentos de Fennema, 4aed., Artmed: Porto Alegre, 2010.

DEMAN, J.M. Principles of food Chemistry. Aspen: Maryland, p.147-149p, 1999.

HE, L.; MU, C.; LI, D.; LIN,W. Revisit the pre-transition of type I collagen denaturation in dilute solution by ultrasensitive differential scanning calorimetry. Thermochim. Acta, v. 548, p.1-5, 2012. 
KAEWDANG, O.; BENJAKUL, S.; KAEWMANEE, T.; KISHIMURA, H. Characteristics of collagens from the swim bladders of yellowfin tuna (Thunnus albacores). Food Chem., v.155, p.264-270, 2014.

LI, D.; MU, C.; CAI, S.; LIN, W. Ultrasonic irradiation in the enzymatic extraction of collagen. Ultrason. Sonochem., v.16, p.605-609, 2009.

LIU, W.; TIAN, Z.; LI, C.; LI, G. Thermal denaturation of fish collagen in solution: A calorimetric and kinetic analysis. Thermochim. Acta, v.581, p.32-40, 2014.

MAXIMO, G.J.; CUNHA, R.L. Mechanical properties of collagen fiber and powder gels. J. Texture Stud. 2010, Doi:10.1111/j.1745-4603.2010.00528.x.

NAGAI, T.; SUZUKI, N.; NAGASHIMA, T. Collagen from common minke whale (Balaenoptera acutotostrata) unesu. Food Chem., 111, p.296-301, 2008.

OCKERMAN, H.W.; HANSEN, C.L. Industrializacíon de subproductos de origen animal. Zaragoza : Acribia, 133 - 160p., 1994.

PATI, F.; ADHIKARI, B.; DHARA, S. Isolation and characterization of fish scale collagen of higher thermal stability. Bioresource Technol., v.101, p.3737-3742, 2010.

ROCHDI, A.; FOUCAT L.; RENOU, J. NMR and DSC studies during thermal denaturation of collagen. Food Chem., v.69, p.295-299, 2000.

SANTANA, R.C.; SATO, A.C.K.; CUNHA, R.L. Emulsions stabilized by heat-treated collagen fibers. Food Hydrocolloid., v.26, p.73-81, 2012.

SCHMIDT, C.G.; SALLAS-MELLADO, M. Influência da ação das enzimas alcalase e flavourzyme no grau de hidrólise das proteínas de carne de frango. Quim. Nova, v.32, n.5, p.1144-1150, 2009.

SCHRIEBER, R.; GAREIS, H. Gelatine Handbook: Theory and Industry Practice. Hardcover, 371p. 2007.

SHIMOKOMAKI, M.; OLIVO, R.; TERRA, N.N.; FRANCO, B.D.G.M. Atualidades em Ciência e Tecnologia de Carnes, São Paulo: Varela, 2006, 236p.

SINTHUSAMRAN, S.; BENJAKUL, S.; KISHIMURA, H. Comparative study on molecular characteristics of acid soluble collagens from skin and swim bladder of seabass (Lates calcarifer). Food Chem., v.138, p.2435-2441, 2013.

TORLEY, P.J.; D'ARCY, B.R.; TROUT, G.R. The effect of ionic strength polyphosphates type, $\mathrm{pH}$, cooking temperature and preblending on the functional properties of normal and pale, soft, exudative (PSE) pork. Meat Sci., v.55, p.451462, 2000.

VEERURAJ, A.; ARUMUGAM, M.; BALASUBRAMANIAN, T. Isolation and characterization of thermostable collagen from the marine eel-fish (Evenchelys macrura). Process Biochem., v.48, p.1592-1602, 2013.

ZHAO, Y.H.; CHI, Y.J. Characterization of collagen from eggshell membrane. Biotechnol., v. 8, n.2, 254-258, 2009. 
WANG, L.; LIANG, Q.; WANG, Z.; XU, J.; LIU, Y.; MA, H. Preparation and characterization of type I collagens from skin of Amur sturgeon (Acipenser schrenckii). Food Chem., v.148, p.410-414, 2014.

WONG, D.W.S. Química de los alimentos: mecanismos y teoría. Zaragoza: Zaragoza, 109-116p, 1995. 